\title{
Atypical Burkitt/Burkitt-Like Lymphoma
}

National Cancer Institute

\section{Source}

National Cancer Institute. Atypical Burkitt/Burkitt-Like Lymphoma. NCI Thesaurus. Code C6917.

A morphologic variant of Burkitt lymphoma characterized by marked nuclear pleomorphism, abundant apoptotic debris, and the presence of tangible body macrophages. 